\title{
Vascularites à anticorps anticytoplasme des polynucléaires neutrophiles et réanimation : quel pronostic en 2018 ?
}

\author{
Antineutrophil Cytoplasmic Antibodies Vasculitis and Intensive Care: \\ what Is the Prognosis for 2018?
}

\author{
A. Kimmoun - B. Levy \\ Reçu le 9 avril 2018; accepté le 29 mai 2018 \\ (C) SRLF et Lavoisier SAS 2018
}

Les vascularites sont une atteinte inflammatoire, nécrosante, souvent systémique, des vaisseaux artériels et veineux dont la classification se fait en fonction de la taille des vaisseaux atteints, du type histologique et du caractère primitif ou secondaire à une autre pathologie [1]. Les vascularites auto-immunes des petits vaisseaux sont souvent associées à des anticorps anticytoplasme des polynucléaires neutrophiles (ANCA). Les présentations cliniques, histologiques, immunologiques ont des caractéristiques bien définies [2]. Néanmoins, l'incidence, bien que mal précisée dans la littérature médicale, est faible, rendant la connaissance de ce groupe de maladie insuffisante en particulier par les réanimateurs [3]. Pourtant, les patients souffrant de telles pathologies peuvent être amenés à une prise en charge en réanimation au moment du diagnostic, pour une récidive ou lors d'une complication des traitements immunosuppresseurs. De façon simplifiée, la présentation clinique la plus fréquente est le syndrome pneumorénal associant sur le plan cytologique et histologique une hémorragie intra-alvéolaire et une glomérulonéphrite rapidement progressive. Les taux de mortalité sont fonction pour les formes les plus sévères au diagnostic à l'entrée en réanimation, de l'ordre de $20 \%$, et pour les prises en charge des complications thérapeutiques, en particulier infectieuses, jusqu'à plus de $70 \%[4,5]$.

De nombreux travaux ont rapporté de façon systématiquement rétrospective et majoritairement monocentrique la présentation et le devenir en réanimation des patients souffrant de vascularites auto-immunes, plus spécifiquement à ANCA. Dans le tableau 1 sont exposées les principales études. Sans être exhaustif, il se concentre sur les travaux qui se sont intéressés aux vascularites des petits vaisseaux à ANCA dont les

\footnotetext{
A. Kimmoun · B. Levy $(\varangle)$

Service de médecine intensive et réanimation Brabois, CHRU de Nancy, U1116, université de Lorraine,

F-54000 Nancy, France

e-mail : b.levy@chru-nancy.fr
}

présentations cliniques en situation d'urgence, et les prises en charge sont relativement homogènes, facilitant ainsi leurs comparaisons. À la vue du grand nombre de centres impliqués dans les deux études rétrospectives multicentriques et du faible nombre de patients inclus, il est possible de conclure que les patients souffrant de vascularites à ANCA sont rares et ont une faible prévalence d'admission en réanimation (Tableau 1). On notera, plus spécifiquement, que la granulomatose avec polyangéite (anciennement dénommée maladie de Wegener) est prédominante en réanimation et que la granulomatose éosinophilique avec polyangéite (anciennement dénommée syndrome de Churg et Strauss) est particulièrement rare. La polyangéite microscopique est plus fréquente que la granulomatose éosinophilique avec polyangéite. Par ailleurs, les motifs d'admission spécifiques sont constants au cours du temps et se résument principalement aux atteintes respiratoires et rénales. Les études incluant les patients en rechute ou anciennement diagnostiqués ont une incidence plus élevée de sepsis reflétant probablement les complications des traitements immunosuppresseurs au long cours. Les survies en réanimation et hospitalière sont aujourd'hui supérieures à $80 \%$ et probablement à rapporter à l'amélioration des soins de support, mais peut-être aussi à l'instauration bien plus précoce des traitements étiologiques immunosuppresseurs y compris dans les situations cliniques les plus sévères impliquant des défaillances pluriviscérales [5]. Concernant ces traitements, on notera l'augmentation du nombre d'échanges plasmatiques réalisés lors des manifestations sévères de ces vascularites au cours des dernières années bien que cette thérapeutique n'ait pas encore parfaitement démontré son efficacité. Les résultats très prochains de l'étude multicentrique PEXIVAS (NCT00987389, Plasma Exchange and Glucocorticoids for Treatment of Anti-Neutrophil Cytoplasm Antibody [ANCA]-Associated Vasculitis) pourraient nous apporter un éclairage sur la pertinence de cette thérapeutique, même si, là encore, les patients les plus sévères étaient exclus de l'étude. Le rituximab est peu prescrit, mais son efficacité n'a pas été 


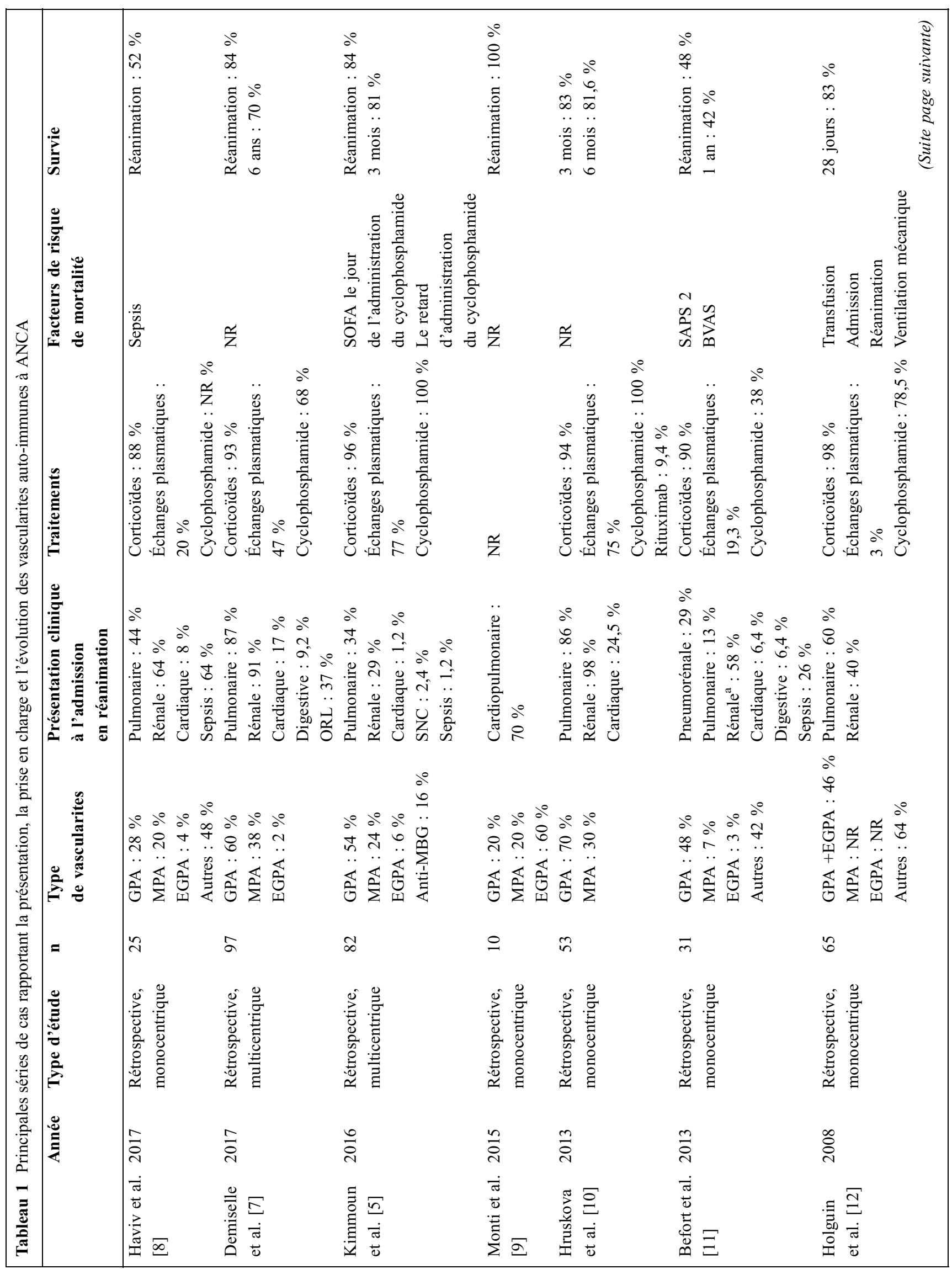




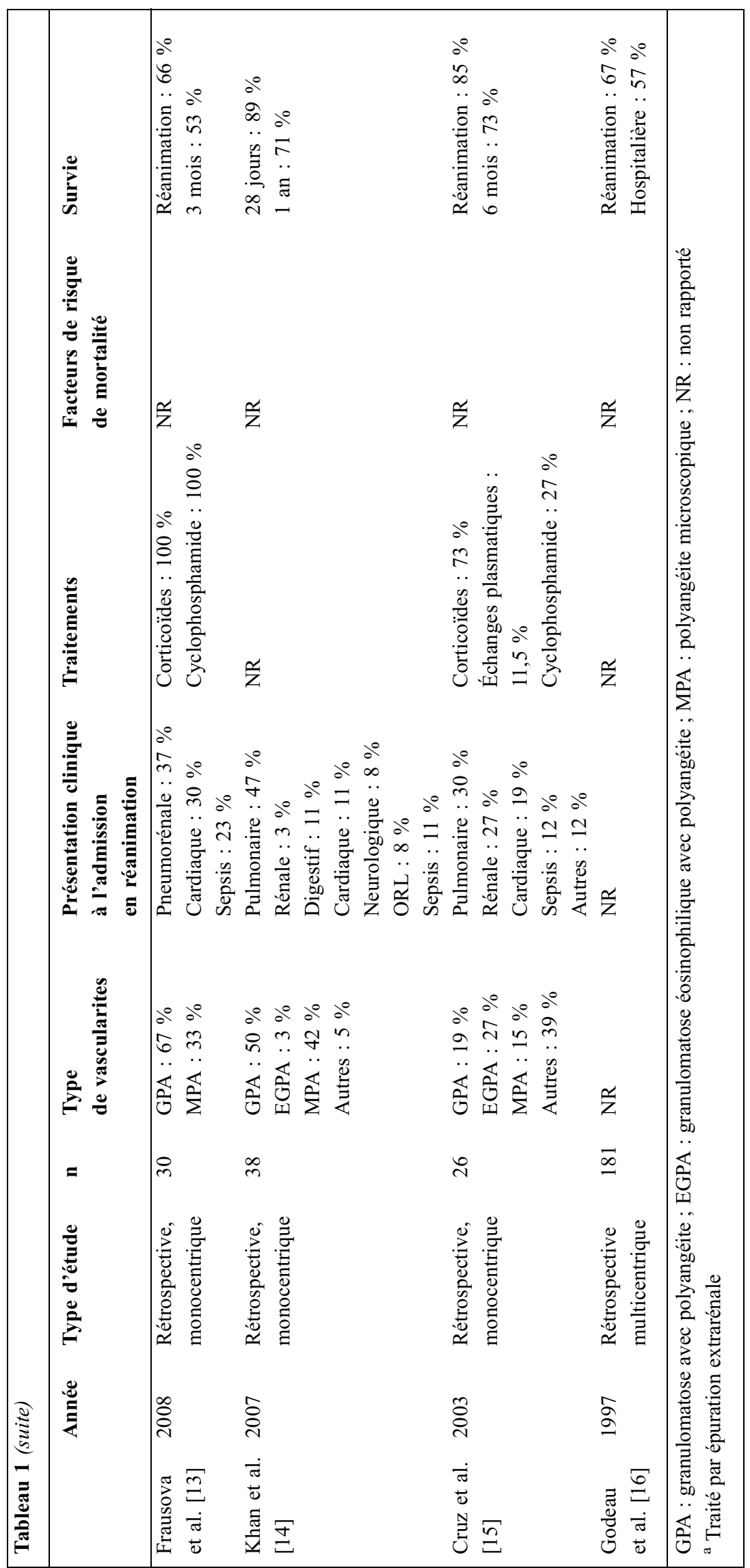


évaluée en réanimation expliquant la prédominance de prescription de cyclophosphamide [6]. Si la mortalité, en diminution, semble essentiellement rapportée à la prise en charge initiale en réanimation, dans les suites, l'amélioration de la survie persiste, puisque jusqu'à $70 \%$ des patients sont survivants à six ans [7].

Finalement, on retiendra, comme souvent en réanimation, qu'un diagnostic rapide et une prise en charge adaptée de ces malades, sous-entendue précoce et complète, incluant les soins de support, les corticoïdes, le cyclophosphamide et, peut-être, les échanges plasmatiques sont associés à un très bon pronostic, y compris à long terme. La nécessité d'une coopération multidisciplinaire incluant les réanimateurs et les médecins spécialistes de ces pathologies doit être la règle.

Liens d'intérêts : les auteurs déclarent ne pas avoir de lien d'intérêt.

\section{Références}

1. Jennette JC, Falk RJ, Andrassy K, Bacon PA, Churg J, Gross WL, Hagen EC, Hoffman GS, Hunder GG, Kallenberg CG, McCluskey RT, Alberto Sinico R, Rees AJ, van Es LA, Whaldheer R, Wiik A, (1994) Nomenclature of systemic vasculitides. Proposal of an international consensus conference. Arthritis Rheum 37: 187-192

2. Semple D, Keogh J, Forni L, Venn R, (2005) Clinical review: vasculitis on the intensive care unit - part 1: diagnosis. Crit Care (London, England) 9: 92-97

3. Mahr A, Guillevin L, Poissonnet M, Ayme S, (2004) Prevalences of polyarteritis nodosa, microscopic polyangiitis, Wegener's granulomatosis, and Churg-Strauss syndrome in a French urban multiethnic population in 2000: a capture-recapture estimate. Arthritis Rheum 51: 92-99

4. Semple D, Keogh J, Forni L, Venn R, (2005) Clinical review: vasculitis on the intensive care unit - part 2: treatment and prognosis. Crit Care (London, England) 9: 193-197

5. Kimmoun A, Baux E, Das V, Terzi N, Talec P, Asfar P, Ehrmann S, Geri G, Grange S, Anguel N, Demoule A, Moreau AS, Azoulay E, Quenot JP, Boisrame-Helms J, Louis G, Sonneville R, Girerd N, Ducrocq N, Agrinier N, Wahl D, Puechal X, Levy B, (2016) Outcomes of patients admitted to intensive care units for acute manifestation of small-vessel vasculitis: a multicenter, retrospective study. Crit Care (London, England) 20: 27

6. Stone JH, Merkel PA, Spiera R, Seo P, Langford CA, Hoffman GS, Kallenberg CG, St Clair EW, Turkiewicz A, Tchao NK,
Webber L, Ding L, Sejismundo LP, Mieras K, Weitzenkamp D, Ikle D, Seyfert-Margolis V, Mueller M, Brunetta P, Allen NB, Fervenza FC, Geetha D, Keogh KA, Kissin EY, Monach PA, Peikert T, Stegeman C, Ytterberg SR, Specks U, Group R-IR, (2010) Rituximab versus cyclophosphamide for ANCA-associated vasculitis. N Engl J Med 363: 221-232

7. Demiselle J, Auchabie J, Beloncle F, Gatault P, Grange S, Du Cheyron D, Dellamonica J, Boyer S, Beauport DT, Piquilloud L, Letheulle J, Guitton C, Chudeau N, Geri G, Fourrier F, Robert R, Guerot E, Boisrame-Helms J, Galichon P, Dequin PF, Lautrette A, Bollaert PE, Meziani F, Guillevin L, Lerolle N, Augusto JF, (2017) Patients with ANCA-associated vasculitis admitted to the intensive care unit with acute vasculitis manifestations: a retrospective and comparative multicentric study. Ann Intensive Care 7: 39

8. Haviv Y, Shovman O, Bragazzi NL, Sharif K, Yavne Y, Shoenfeld Y, Amital H, Watad A, (2018) Patients With Vasculitides Admitted to the Intensive Care Unit: Implications From a Single-Center Retrospective Study. J Intensive Care Med (2017 Jan 1:885066617717223. doi: 10.1177/0885066617717223. [Epub ahead of print])

9. Monti S, Montecucco C, Pieropan S, Mojoli F, Braschi A, Caporali $R$, (2015) Life-threatening onset of systemic vasculitis requiring intensive care unit admission: a case series. Clin Exper Rheumatol 33: S126-S131

10. Hruskova Z, Casian AL, Konopasek P, Svobodova B, Frausova D, Lanska V, Tesar V, Jayne DR, (2013) Long-term outcome of severe alveolar haemorrhage in ANCA-associated vasculitis: a retrospective cohort study. Scand J Rheumatol 42: 211-214

11. Befort P, Corne P, Filleron T, Jung B, Bengler C, Jonquet $\mathrm{O}$, Klouche K, (2013) Prognosis and ICU outcome of systemic vasculitis. BMC Anesthesiol 13: 27

12. Holguin F, Ramadan B, Gal AA, Roman J, (2008) Prognostic factors for hospital mortality and ICU admission in patients with ANCA-related pulmonary vasculitis. Am J Med Sci 336: 321-326

13. Frausova D, Brejnikova M, Hruskova Z, Rihova Z, Tesar V, (2008) Outcome of thirty patients with ANCA-associated renal vasculitis admitted to the intensive care unit. Ren Fail 30: 890-895

14. Khan SA, Subla MR, Behl D, Specks U, Afessa B, (2007) Outcome of patients with small-vessel vasculitis admitted to a medical ICU. Chest 131: 972-976

15. Cruz BA, Ramanoelina J, Mahr A, Cohen P, Mouthon L, Cohen Y, Hoang P, Guillevin L, (2003) Prognosis and outcome of 26 patients with systemic necrotizing vasculitis admitted to the intensive care unit. Rheumatology (Oxford, England) 42: 1183-1188

16. Godeau B, Mortier E, Roy PM, Chevret S, Bouachour G, Schlemmer B, Carlet J, Dhainaut JF, Chastang C, (1997) Short and longterm outcomes for patients with systemic rheumatic diseases admitted to intensive care units: a prognostic study of 181 patients. J Rheumatol 24: 1317-1323 